\title{
VISUO-SPATIAL MEMORY IMPAIRMENT IN OBSESSIVE COMPULSIVE DISORDER (OCD): FROM NEUROPSYCHOLOGICAL FINDINGS TO EYE-TRACKING
}

\author{
Gyula Demeter ${ }^{1}$, Péter Pajkossy ${ }^{1}$, Ágnes Szőllősi ${ }^{1}$, András Harsányi ${ }^{2}$, Katalin Csigó ${ }^{2}$, Mihály Racsmány ${ }^{1}$ \\ ${ }^{1}$ Department of Cognitive Science, Budapest University of Technology and Economics, Hungary \\ ${ }^{2}$ Department of Psychiatry II., Nyírő Gyula Hospital, National Institute of Psychiatry and Addictions, \\ Budapest, Hungary
}

There is a growing amount of evidence about the deficit of executive system in OCD, which is strongly related to the fronto-basal loop dysfunctions of the disorder (e.g. Chamberlain et al., 2005; Olley et al., 2007). In addition to the executive deficit, several studies found impaired visual and spatial memory performance in this disorder. In an early neuropsychological model, Savage (1998) suggested that the fronto-striatal dysfunctions along with impaired executive functioning leads to observed memory difficulties (encoding and retrieval of nonverbal information). Following this suggestion, here we aimed at investigating visuo-spatial memory functions in a pool of OCD patients in two studies. We combined neuropsychological tasks and computer based working memory paradigms with eye-tracking methodology. In Study 1 the Rey Complex Figure Task (RCFT) was used and it was found that OCD patients performed significantly poorer in the copy and recall phase of the task in comparison with matched healthy control group. Symptom severity and the level of anxiety were assessed by the Yale Brown Obsessive Compulsive Scale (Y-BOCS) and the State-Trait Anxiety Inventory (STAI), respectively. There was a significant negative correlation between symptom severity (Y-BOCS total scores) and the recall scores of the RCFT. In Study 2 we developed a computer-based Visual Pattern Task to investigate the eye movement correlates of cognitive processes involved in visuo-spatial working memory function. In addition subjects were also screened by a neuropsychological test battery including tasks on shifting (Wisconsin Card Sorting Test), response inhibition (Stop Signal Task, Stroop Task) and working memory capacity functions (n-back Task). According to our preliminary eye-tracking results, in comparison with healthy control subjects, OCD patients produced a significant decrease in target-related dwell time scores during the delay phase of the task. We will discuss the possible relationships between eye movement patterns, cognitive deficits and symptom severity.

Keywords: visuo-spatial memory, eye-tracking, OCD

Financial support for the research was provided by KTIA_NAP_13 (Neurocognitive disorders of frontostriatal system) and OTKA (Hungarian National Science Foundation) PD 112502. Gyula Demeter was supported by the János Bolyai Research Scholarship of the Hungarian Academy of Sciences.

The authors declare that there are no conflicts of interest. 